\title{
Antihypertensive Effectiveness of Perindopril Arginine and Indapamide Single-Pill Combination According to Body Mass Index: Findings from the FORSAGE Study
}

\author{
M. G. Glezer • The FORSAGE Investigators
}

Received: October 10, 2019 / Published online: February 1, 2020

(C) The Author(s) 2020

\begin{abstract}
Introduction: Overweight and obesity are increasing worldwide and are associated with an increased risk for cardiovascular disease (CVD). The aim of this study was to examine the burden of CVD risk factors among normal weight, overweight, and obese subjects with hypertension, and to evaluate the effectiveness of switching to a single-pill combination (SPC) of perindopril arginine/indapamide for blood pressure (BP) control in overweight and obese subjects treated in routine clinical practice.

Methods: FORSAGE was a 3-month, multicenter, observational, open-label study conducted in Russian patients with uncontrolled arterial hypertension under previous antihypertensive therapy. Subjects were switched to the full-dose
\end{abstract}

Enhanced Digital Features To view enhanced digital features for this article go to https://doi.org/10.6084/ m9.figshare.11603973.

For a list of study investigators see Online Supplementary Material.

Electronic supplementary material The online version of this article (https://doi.org/10.1007/s40119020-00162-x) contains supplementary material, which is available to authorized users.

M. G. Glezer $(\bowtie)$

Department of Preventive and Emergency

Cardiology, Sechenov First Moscow State Medical

University, Moscow, Russia

e-mail: 287ast@mail.ru perindopril arginine $10 \mathrm{mg} /$ indapamide $2.5 \mathrm{mg}$ SPC. BP was assessed at 2 weeks, 1 month, and 3 months, and serum creatinine and general health status at 3 months. The present post hoc analysis of the FORSAGE study results explored the effectiveness of perindopril arginine/indapamide SPC in patients with arterial hypertension with regard to baseline body mass index (BMI): normal $\left(<25 \mathrm{~kg} / \mathrm{m}^{2}\right)$, overweight $\left(25 \leq \mathrm{BMI}<30 \mathrm{~kg} / \mathrm{m}^{2}\right)$, and obese $(\geq 30 \mathrm{~kg} /$ $\mathrm{m}^{2}$ ).

Results: A total of 1969 patients were recruited, but BMI data were available for 1963 patients, two-thirds of whom were women. The distribution of BMI groups was as follows: $<25 \mathrm{~kg} /$ $\mathrm{m}^{2}(16.7 \%)$, overweight $(48.7 \%)$, and obese $(34.7 \%)$. Overweight or obese patients had more concomitant diseases such as diabetes mellitus or history of stroke, higher BP levels, serum cholesterol and creatinine, and lower glomerular filtration rates. Switching to perindopril arginine/indapamide SPC was associated with a statistically significant reduction in BP as early as the second week of treatment. At 3 months, systolic blood pressure (SBP)/diastolic blood pressure (DBP) had decreased significantly by $39.3 / 18.8 \mathrm{mmHg}$ in the normal BMI group, $39.8 / 18.8 \mathrm{mmHg}$ in overweight, and 39.4/ $18.7 \mathrm{mmHg}$ in obese groups. The magnitude of the $\mathrm{BP}$ reduction was independent of BMI. Achievement of target BP $(<140 / 90 \mathrm{mmHg})$ was good in all groups, but lower in obese subjects $(70.9 \%)$ than in overweight subjects 
(78.1\%) or those with a normal BMI $(81.8 \%)$ ( $P<0.0001$ for both comparisons).

Conclusions: In subjects with uncontrolled BP on existing antihypertensive therapy, switching to perindopril arginine $10 \mathrm{mg}$ /indapamide $2.5 \mathrm{mg}$ was associated with statistically significant decreases in BP and higher rates of target BP achievement in all BMI groups, including more than $70 \%$ of overweight and obese patients.

Trial Registration: ISRCTN ID, ISRCTN14 315146 (retrospectively registered 18/11/2019).

Keywords: Blood pressure target; Body mass index; Indapamide; Obesity; Perindopril arginine; Quality of life; Single-pill combinations

\section{Key Summary Points}

\section{Why carry out this study?}

Rates of blood pressure control among overweight and obese individuals are often suboptimal due to specific pathogenetic mechanisms and additional comorbidities and risk factors.

A post hoc analysis of the observational FORSAGE study was conducted to assess the effectiveness of switching patients on previous antihypertensive therapy to a perindopril arginine/indapamide $(10 \mathrm{mg} /$ $2.5 \mathrm{mg}$ ) single-pill combination with regard to baseline body mass index (BMI).

\section{What was learned from the study?}

High rates of target blood pressure achievement were observed in all BMI groups including the overweight and obese.

The mechanisms of action of perindopril and indapamide may make them particularly suitable for use in obesityassociated hypertension.

\section{INTRODUCTION}

The achievement of blood pressure (BP) targets is dependent on many factors, including adequate treatment prescription, patient adherence to treatment, as well as the characteristics of the patient and the presence of comorbidities [1]. In particular, suboptimal efficacy of antihypertensive therapy is often found in patients with obesity, type 2 diabetes mellitus, target organ damage, or hypertension-related diseases such as coronary artery disease [2-6].

One method to improve the effectiveness of treatment is to increase the number of prescribed antihypertensive agents and their doses [7], preferably in the form of a single-pill combination (SPC).

In 2015, we conducted the open-label, observational FORSAGE study in which patients were switched from previous antihypertensive therapy to full-dose perindopril arginine/indapamide SPC and physicians educated on how to encourage patients to lead a healthy lifestyle with the aim of improving achievement of target BP and increasing adherence to treatment [8]. The data obtained from FORSAGE indicated that switching to full-dose perindopril arginine/ indapamide SPC resulted in three quarters of the study population achieving target BP values [8]. Moreover, the increase in treatment effectiveness was similar between the group of patients whose physicians received additional education within the program and those who did not [8].

Nevertheless, almost a quarter of patients did not achieve the target BP level. The main factors underlying this failure were male gender, age over 60 years, poor treatment adherence at baseline, persistent high cholesterol levels, higher baseline BP levels, low glomerular filtration rate (GFR), high heart rate, and increased body mass index (BMI) [8]. The aim of the current post hoc analysis of the FORSAGE study was to assess the effectiveness of the perindopril arginine/indapamide $(10 \mathrm{mg} / 2.5 \mathrm{mg})$ SPC in patients with arterial hypertension with regard to baseline BMI. 


\section{METHODS}

FORSAGE was a 3-month, multicenter, openlabel, observational study carried out in 29 cities in the Russian Federation from November 2014 to April 2016. Patients were randomized into three groups. In group 1, patients were followed up by the physician according to routine clinical practice without any specific recommendations. In group 2, physicians received training on current guidelines for the management of hypertension and in effective patient communication for increasing treatment adherence. In group 3, physicians received training on current guidelines for the management of hypertension and effective patient communication, and patients were trained in self-monitoring of BP. Patients in groups 2 and 3 also received counseling for non-pharmacological treatments for hypertension, including dietary and salt intake recommendations. The methods and main findings of the study have been previously published in Russian [8]. A total of 442 general practitioners (GPs) and internists participated in the program and enrolled 1969 patients with essential arterial hypertension having BP $>150$ / $90 \mathrm{mmHg}$ despite treatment. Inclusion criteria were: age over 18 years and $\mathrm{BP}>150 / 90 \mathrm{mmHg}$ on current antihypertensive therapy. Treatment with perindopril/indapamide SPC was based on physician's judgment. Perindopril arginine/indapamide $(10 \mathrm{mg} / 2.5 \mathrm{mg})$ was administered in accordance with clinical situation and the drug label. The dose of perindopril arginine/indapamide $(10 \mathrm{mg} / 2.5 \mathrm{mg})$ could be decreased to perindopril arginine/indapamide $(5 \mathrm{mg} /$ $1.25 \mathrm{mg}$ ) in case of need.

Non-inclusion criteria were as follows: secondary hypertension, pregnancy, breast-feeding, severe cardiovascular disease (CVD; acute myocardial infarction or acute cerebrovascular accident within the last 6 months), diabetes mellitus in the stage of decompensation, severe hepatic impairment, renal transplantation, nephrectomy or the presence of a single kidney, hypokalemia or hyperkalemia, chronic alcoholism, abuse of drugs, contraindications, intolerance or hypersensitivity reactions to perindopril arginine/indapamide or its individual components, systemic connective tissue diseases, aortic stenosis, malignant neoplasm, presence of indapamide $1.25 \mathrm{mg} /$ perindopril $5 \mathrm{mg}$ or indapamide $2.5 \mathrm{mg} /$ perindopril $10 \mathrm{mg}$ or free combination of its compounds (perindopril/indapamide) in a current therapy, inability to understand the essence of the study and to give valid consent to participate.

Data were collected during four visits to participating centers at the following intervals: baseline, 2 weeks, 1 month (M1), and 3 months (M3). Investigators collected patient demographic data as well as information on risk factors, history of cardiovascular events, BP, resting heart rate, and current cardiovascular treatments. As this was an observational study, no unified echocardiographic methods were specified, and both 2D Doppler and M-mode methods could be used. Data on systolic and diastolic BP (SBP and DBP) and heart rate were collected at all four visits; serum creatinine levels were assessed at baseline and M3. General health status was assessed at baseline and M3 using the visual analogue scale (VAS) scored from 0 to 100 points.

All patients meeting the study inclusion criteria were switched to perindopril/indapamide SPC $(10 \mathrm{mg} / 2.5 \mathrm{mg})$. During the study, angiotensin-converting enzyme inhibitors (ACEI), angiotensin receptor blockers (ARB), and diuretics could be replaced by perindopril/indapamide SPC. If a patient was also receiving therapy from other classes of antihypertensive drugs, this therapy could be continued.

Patients with a known BMI $(n=1963)$ were divided into three groups according to their baseline value as follows: (1) normal BMI $\left(<25 \mathrm{~kg} / \mathrm{m}^{2}\right)$, (2) overweight $(25 \leq \mathrm{BMI}<30 \mathrm{~kg} /$ $\left.\mathrm{m}^{2}\right)$, and (3) obese (BMI $\left.\geq 30 \mathrm{~kg} / \mathrm{m}^{2}\right)$.

Treatment effectiveness was assessed based on change in BP and rate of target BP achievement (for patients who completed the program in accordance with the protocol) at 2 weeks, M1, and M3. Adverse events were monitored throughout the study. 


\section{Statistical Analysis}

Data were analyzed using SAS statistical software version 6.12 and are presented as mean values, standard deviation, standard error of the mean, minimum and maximum values, and median with quintiles for continuous variables, or as absolute number and relative frequency of occurrence of each possible value for qualitative or categorical variables. Values before and after treatment for the whole population and BMI subgroups were compared using Student's $t$ test (or Fisher's exact test) for paired observations, or non-parametric Wilcoxon, Mann-Whitney, and chi-square tests. Treatment subgroups were compared using analysis of variance. $P<0.05$ was considered statistically significant.

\section{Compliance with Ethics Guidelines}

The program was approved at the meeting of the Inter-University Ethical Committee, Protocol No. 06-14 dated 20.06.2014 (Moscow). The study was performed in accordance with the International Council for Harmonisation of Technical Requirements for Pharmaceuticals for Human Use (ICH) guidelines for good clinical practice and the ethical principles derived from the revised Declaration of Helsinki. All patients provided written informed consent.

\section{RESULTS}

Of the 1969 patients in the FORSAGE study, 1963 had baseline BMI data available and were included in the present analysis. A total of 32 patients $(1.6 \%)$ withdrew from the study prematurely: nine were lost to follow-up, 11 declined to participate, four discontinued due to the doctor's decision, two withdrew due to lack of efficacy and six for adverse events. Demographic and baseline clinical characteristics are shown in Table 1 . The mean age was $60.1 \pm 0.3$ years, and $64.3 \%$ were women. A normal BMI was observed in $16.7 \%$ of patients, almost half were overweight (48.7\%), and over one-third were obese $(34.7 \%)$. The mean BMI values in the three groups were $23.3 \mathrm{~kg} / \mathrm{m}^{2}$, $27.4 \mathrm{~kg} / \mathrm{m}^{2}$, and $33.6 \mathrm{~kg} / \mathrm{m}^{2}$, respectively.

The proportions of patients receiving the different types of antihypertensive drugs are described in Table 2. At study entry, 1682 patients $(85.7 \%)$ were receiving free combinations of antihypertensive drugs, and 287 $(14.6 \%)$ were receiving fixed combinations. The single-pill combinations were stopped when patients started treatment with perindopril/indapamide SPC. The proportion of patients receiving concomitant treatment after having started perindopril/indapamide SPC decreased during visits $1-3$. At visit $3,32.4 \%$ of patients were receiving beta blockers vs. $41.6 \%$ at visit 0 , $12.0 \%$ of patients were receiving CCB vs. $25.9 \%$ at visit $0,1.8 \%$ were receiving diuretics other than indapamide vs. $35.4 \%$ receiving diuretics (including indapamide) at visit 0 , and $0.6 \%$ of patients were receiving ARB vs. $26.0 \%$ at visit 0 .

No significant difference was observed between the mean number of drugs taken by patients with normal BMI (3.9) and by overweight subjects (4.1), but obese patients were using statistically more drug classes (4.4; $P=0.0001$ ).

At baseline, a number of differences in demographic and clinical characteristics were observed between the three BMI groups (Table 1). In all groups, there was a higher proportion of women than men, but particularly in the obese group (72.1\%). SBP and DBP values were high in all groups, and small but statistically significant increases in BP were observed in both the overweight and obese groups compared with the normal BMI group. Small but statistically significant increases in mean total cholesterol levels were also observed in both the overweight and obese groups compared with normal BMI and between the overweight and obese groups. The proportions of patients with hypercholesterolemia (total cholesterol $>5 \mathrm{mmol} / \mathrm{L}$ ) were higher in the overweight and obese BMI groups compared with patients with normal BMI. Obese subjects were found to have statistically higher serum creatinine concentrations and lower GFR compared with the other groups.

The proportion of patients with type 2 diabetes was statistically greater in both 
Table 1 Patient characteristics according to age-adjusted body mass index at baseline

\begin{tabular}{|c|c|c|c|c|c|c|c|}
\hline Parameter & $\begin{array}{l}\text { Total } \\
\text { group }\end{array}$ & $\begin{array}{l}\text { Normal } \\
\text { BMI group }\end{array}$ & $\begin{array}{l}\text { Overweight } \\
\text { group }\end{array}$ & $\boldsymbol{P}$ & $\begin{array}{l}\text { Obese } \\
\text { group }\end{array}$ & $\boldsymbol{P}$ & $P 1$ \\
\hline Number of patients (\%) & $1963(100)$ & $327(16.7)$ & $955(48.7)$ & & $681(34.7)$ & & \\
\hline Mean age $( \pm S D)$, years & $60.1 \pm 0.3$ & $59.4 \pm 0.6$ & $59.2 \pm 0.3$ & NS & $60.3 \pm 0.40$ & NS & $<0.05$ \\
\hline Male, $n(\%)$ & $702(35.7)$ & $120(36.7)$ & $392(41.1)$ & & $190(27.9)$ & & \\
\hline Female, $n(\%)$ & $1261(64.3)$ & $207(63.3)$ & $563(58.9)$ & & $491(72.1)$ & & \\
\hline Current smoker, $n(\%)$ & $434(22.0)$ & $96(29.3)$ & $278(29.1)$ & NS & $172(25.2)$ & NS & $<0.05$ \\
\hline $\begin{array}{l}\text { History of hypertension in one } \\
\text { parent, } n(\%)\end{array}$ & $1064(54.0)$ & $181(55.4)$ & $525(55.0)$ & NS & $371(54.4)$ & NS & NS \\
\hline $\begin{array}{l}\text { History of hypertension in } \\
\text { both parents, } n(\%)\end{array}$ & $497(25.2)$ & $68(20.8)$ & $243(25.4)$ & NS & $193(28.4)$ & NS & $<0.05$ \\
\hline $\begin{array}{l}\text { Mean duration of } \\
\text { hypertension }( \pm S D) \text {, years }\end{array}$ & $10.3 \pm 0.9$ & $11.3 \pm 1.6$ & $10.1 \pm 0.9$ & NS & $10.3 \pm 0.9$ & NS & NS \\
\hline \multicolumn{8}{|l|}{ Medical history, n (\%) } \\
\hline Angina pectoris & $514(26.1)$ & $78(23.9)$ & $250(26.2)$ & NS & $210(30.8)$ & 0.05 & $<0.05$ \\
\hline Myocardial infarction & $129(6.6)$ & $28(8.8)$ & $75(7.9)$ & NS & $55(8.1)$ & NS & NS \\
\hline Stroke & $129(6.6)$ & $19(5.9)$ & $54(5.6)$ & NS & $57(8.4)$ & NS & $<0.05$ \\
\hline Type 2 diabetes & $276(14.0)$ & $23(7.1)$ & $113(11.8)$ & $<0.05$ & $152(22.3)$ & 0.0001 & 0.0001 \\
\hline Heart failure & $1442(73.2)$ & $246(75.2)$ & $683(71.5)$ & NS & $530(77.9)$ & NS & $<0.01$ \\
\hline Body mass index, $\mathrm{kg} / \mathrm{m}^{2}$ & $28.4 \pm 0.2$ & $23.3 \pm 0.1$ & $27.4 \pm 0.1$ & 0.0001 & $33.6 \pm 0.1$ & 0.0001 & 0.0001 \\
\hline Systolic BP, mmHg & $169.1 \pm 1.8$ & $168.1 \pm 0.5$ & $169.8 \pm 0.3$ & $<0.05$ & $171.2 \pm 0.3$ & 0.001 & NS \\
\hline Diastolic BP, mmHg & $98.5 \pm 1.3$ & $97.3 \pm 0.3$ & $98.1 \pm 0.2$ & $<0.05$ & $98.8 \pm 0.3$ & 0.0001 & $<0.05$ \\
\hline Heart rate, bpm & $77.2 \pm 0.6$ & $76.7 \pm 0.5$ & $77.0 \pm 0.3$ & NS & $77.8 \pm 0.4$ & NS & NS \\
\hline Creatinine, $\mu \mathrm{mol} / \mathrm{L}$ & $87.9 \pm 0.7$ & $85.6 \pm 0.4$ & $85.7 \pm 0.3$ & NS & $88.1 \pm 0.3$ & $<0.0001$ & 0.0001 \\
\hline $\begin{array}{l}\text { CKD-EPI glomerular } \\
\text { filtration rate, } \mathrm{mL} / \mathrm{min} / \\
1.73 \mathrm{~m}^{2}\end{array}$ & $73.7 \pm 0.9$ & $73.9 \pm 2.7$ & $74.3 \pm 1.5$ & NS & $69.4 \pm 0.9$ & 0.0001 & 0.0001 \\
\hline Total cholesterol, $\mathrm{mmol} / \mathrm{L}$ & $5.7 \pm 0.1$ & $5.6 \pm 0.06$ & $5.8 \pm 0.03$ & $<0.05$ & $5.9 \pm 0.04$ & $<0.001$ & $<0.05$ \\
\hline $\begin{array}{l}\text { Number of patients with TC } \\
\text { concentration more than } \\
5 \mathrm{mmol} / \mathrm{L}, n(\%)\end{array}$ & $1490(75.9)$ & $221(67.5)$ & $732(76.6)$ & $<0.01$ & $537(78.9)$ & $<0.0001$ & NS \\
\hline ECG signs of LVH, $n(\%)$ & $1550(78.7)$ & $245(74.9)$ & $752(78.8)$ & NS & $581(85.3)$ & $<0.001$ & $<0.0001$ \\
\hline $\begin{array}{l}\text { Echocardiographic signs }{ }^{\mathrm{a}} \text { of } \\
\text { LVH, } n(\%)\end{array}$ & $1328(67.4)$ & $204(62.5)$ & $650(68.1)$ & NS & $486(71.4)$ & $<0.01$ & NS \\
\hline $\begin{array}{l}\text { ECG and echocardiographic } \\
\text { signs }^{\mathrm{a}} \text { of LVH, } n(\%)\end{array}$ & $1210(61.5)$ & $190(58.2)$ & $584(61.2)$ & NS & $455(66.8)$ & $<0.01$ & $<0.05$ \\
\hline
\end{tabular}


Table 1 continued

\begin{tabular}{|c|c|c|c|c|c|c|c|}
\hline Parameter & $\begin{array}{l}\text { Total } \\
\text { group }\end{array}$ & $\begin{array}{l}\text { Normal } \\
\text { BMI group }\end{array}$ & $\begin{array}{l}\text { Overweight } \\
\text { group }\end{array}$ & $P$ & $\begin{array}{l}\text { Obese } \\
\text { group }\end{array}$ & $P$ & $P 1$ \\
\hline Hypertensive crisis, $n$ (\%) & $625(31.7)$ & $92(27.1)$ & $295(30.9)$ & NS & $241(35.4)$ & $<0.01$ & NS \\
\hline $\begin{array}{l}\text { Hospital admissions for } \\
\text { hypertensive crisis, } n \text { (\%) }\end{array}$ & $305(15.5)$ & $28(8.6)$ & $158(16.5)$ & $<0.001$ & $124(18.2)$ & $<0.0001$ & NS \\
\hline VAS score & $46.2 \pm 2.0$ & $46.7 \pm 0.7$ & $46.9 \pm 0.4$ & NS & $45.5 \pm 0.5$ & NS & NS \\
\hline
\end{tabular}

Values are mean \pm SE or numbers and corresponding percentages

$P$, comparison between overweight or obese vs. normal BMI group

$P 1$, comparison between obese vs. overweight group

$B M I$ body mass index, $H T$ hypertension, NYHA New York Heart Association, BP blood pressure, CKD-EPI formula for calculation of glomerular filtration rate, $L V H$ left ventricular hypertrophy, $E C G$ electrocardiography, $E c h o C G$ echocardiography, $V A S$ visual analogue scale, $N S$ non-significant

${ }^{a}$ Echocardiographic LVH could be defined using both M-mode and 2D Doppler methods

Table 2 The proportion of patients receiving major antihypertensive drug classes at baseline

\begin{tabular}{ll}
\hline $\begin{array}{l}\text { Class of } \\
\text { antihypertensive } \\
\text { drugs }\end{array}$ & $\begin{array}{l}\text { Number (\%) of patients on free } \\
\text { combinations }\end{array}$ \\
\hline ACEI & $866(51.5)$ \\
ARB & $437(26.0)$ \\
CCB & $436(25.9)$ \\
Diuretics & $595(35.4)$ \\
BB & $699(41.6)$ \\
Alpha-blockers & $8(0.5)$ \\
& Number (\%) of patients on single- \\
ACEI + diuretics & $177(61.5)$ \\
ARB + diuretics & $56(19.6)$ \\
ACEI + CCB & $41(14.3)$ \\
ARB + CCB & $13(4.5)$ \\
\hline
\end{tabular}

$A C E I$ angiotensin-converting enzyme inhibitors, $A R B$ angiotensin receptor blocker, $B B$ beta blocker, $C C B$ calcium channel blockers overweight and obese groups compared with the normal BMI group, and also differed between the obese and overweight groups (22.3 vs. $11.8 \%$, respectively, $P=0.0001$; Table 1 ). Obese patients had higher occurrence rates of left ventricular hypertrophy, exertional angina pectoris, and heart failure, and were more likely to have a history of stroke, hypertensive crisis, or hospitalization for hypertensive crisis. Overweight patients had a higher rate of hospitalization for hypertensive crisis compared with patients with normal BMI.

There were no significant differences in duration of hypertension or heart rate between groups, and no differences in well-being estimates.

At baseline, SBP levels were higher in overweight and obese patients than patients with normal BMI, and SBP was significantly higher in obese patients than in overweight patients (Table 3). Switching to a perindopril arginine/ indapamide SPC was associated with a significant reduction in SBP in all BMI groups (Fig. 1). At each visit, SBP levels were significantly lower than at the previous one $(P=0.0001)$. Although the absolute SBP values remained slightly higher in obese and overweight patients compared with patients with normal BMI, patients in all groups achieved the target SBP 
Table 3 Blood pressure (BP) levels before and during treatment with perindopril arginine/indapamide single-pill combination according to baseline body mass index $(\mathrm{BMI})$

\begin{tabular}{|c|c|c|c|c|c|c|}
\hline Parameter & Normal BMI group & Overweight group & $P$ & Obese group & $P$ & $P 1$ \\
\hline \multicolumn{7}{|c|}{ Systolic BP, mmHg } \\
\hline Baseline & $168.1 \pm 0.5$ & $169.8 \pm 0.3$ & 0.001 & $171.2 \pm 0.3$ & $<0.001$ & 0.0001 \\
\hline 2 weeks & $146.2 \pm 0.5$ & $147.0 \pm 0.3$ & NS & $148.1 \pm 0.3$ & 0.0001 & $<0.05$ \\
\hline 1 month & $135.2 \pm 0.5$ & $136.6 \pm 0.3$ & $<0.05$ & $138.3 \pm 0.3$ & 0.0001 & 0.0001 \\
\hline 3 months & $128.8 \pm 0.5$ & $130.0 \pm 0.3$ & $<0.05$ & $131.8 \pm 0.3$ & 0.0001 & 0.0001 \\
\hline \multicolumn{7}{|c|}{ Diastolic BP, mmHg } \\
\hline Baseline & $97.3 \pm 0.3$ & $98.1 \pm 0.2$ & $<0.05$ & $98.8 \pm 0.2$ & 0.0001 & $<0.05$ \\
\hline 2 weeks & $86.7 \pm 0.3$ & $87.3 \pm 0.2$ & NS & $87.9 \pm 0.2$ & $<0.05$ & NS \\
\hline 1 month & $81.6 \pm 0.3$ & $82.3 \pm 0.2$ & NS & $83.3 \pm 0.2$ & 0.0001 & $<0.001$ \\
\hline 3 months & $78.5 \pm 0.3$ & $79.3 \pm 0.2$ & $<0.05$ & $80.1 \pm 0.2$ & 0.0001 & $<0.01$ \\
\hline
\end{tabular}

Data presented are mean \pm SE

$P$, comparison between overweight or obese vs. normal BMI group

$P 1$, comparison between obese vs. overweight group

of $<140 \mathrm{mmHg}$ at M3 despite differences in cardiovascular risk profiles and the target level of $\mathrm{BP}$ in patients in comparison groups.

While absolute DBP values remained higher in obese patients at all visits compared with patients with normal BMI and overweight patients, a continuous reduction in DBP levels was observed at each visit in all groups $(P=0.0001)$ (Fig. 1). In overweight patients, differences in DBP reached statistical significance compared with patients with normal BMI at M3.

After 3 months of treatment with the singlepill combination, over $70 \%$ of patients had achieved target BP levels $(<140 / 90 \mathrm{~mm} \mathrm{Hg})$ in all three groups (Table 4 ). The proportion of patients achieving BP target levels decreased with increasing BMI level. At 2 weeks, the proportion of patients who achieved the target BP was similar in all three groups (Table 4), but at M1 and M3 the proportions of overweight and especially obese patients who achieved target BP were significantly lower than in the group with normal BMI.

Creatinine levels had decreased in all groups at M3, although they remained higher in obese patients compared with the other two BMI groups $(P=0.001)$ (Table 5). Over the course of the study, creatinine clearance increased in all groups, even in the obese, with no statistical differences between groups at M3 (Table 5).

During the study, adverse events were reported in seven patients $(0.36 \%$ of the included patients). Five patients experienced dry cough, one of them with associated insomnia. One patient experienced pruritus and insomnia. All these cases were assessed as related to the drug and not serious, and all patients recovered after drug discontinuation. One serious adverse event was reported. This concerned a 52-yearold woman who experienced clinically manifest hypotension (BP 100/60 mm Hg). The patient completed the study as per protocol, but further action regarding the drug and outcome are unknown. The case was not reported as serious, but it was upgraded in seriousness by the Therapeutic Safety Department.

Two patients stopped their participation in the study due to a lack of treatment efficacy. Information about outcome was not available for these patients. 

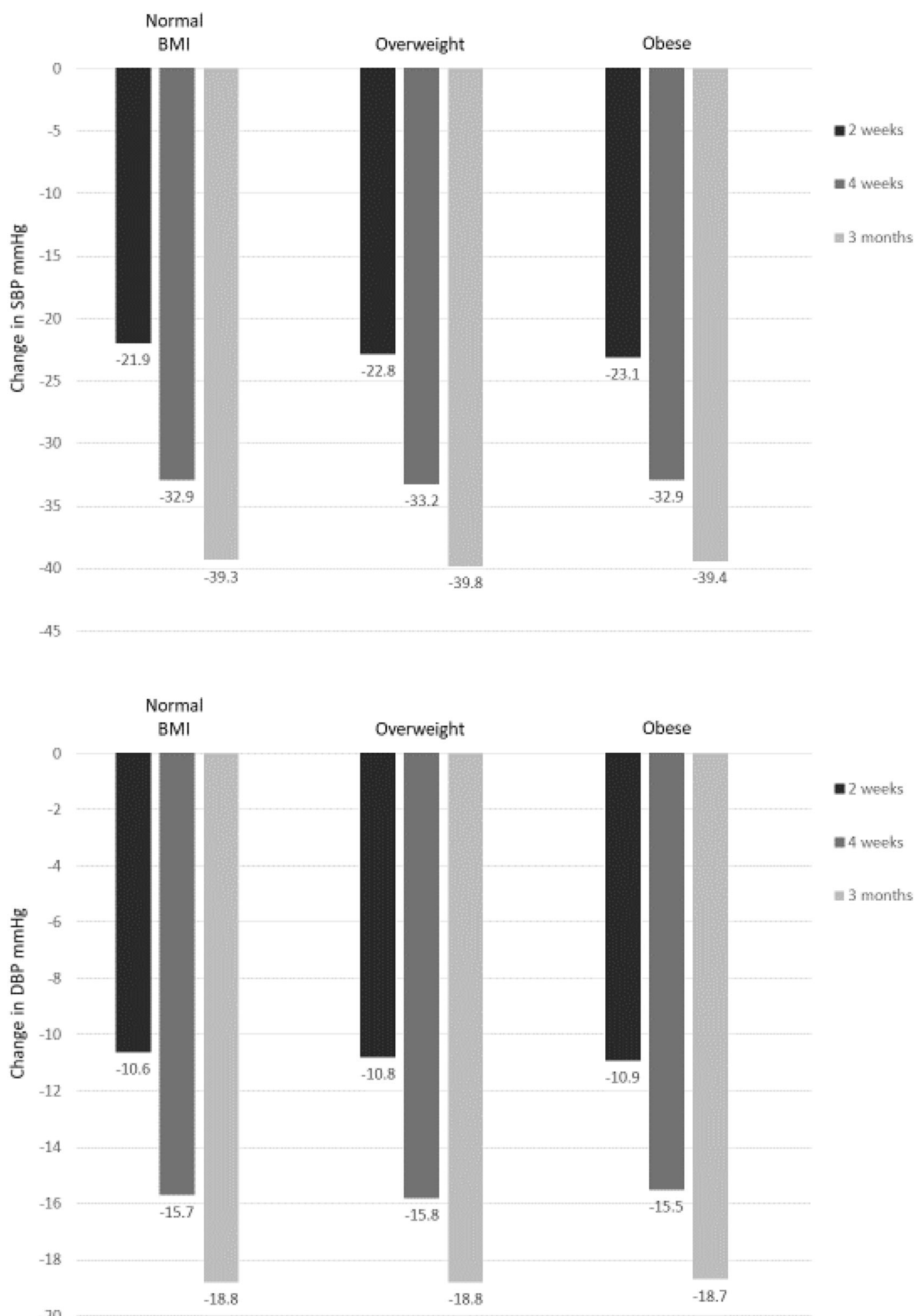

Fig. 1 Change in mean systolic (SBP) and diastolic blood pressure (DBP) levels at each study visit compared with baseline during treatment with perindopril arginine/ indapamide single-pill combination according to baseline body mass index (BMI) group 
Table 4 Proportion of patients (\%) achieving the target blood pressure level $(<140 / 90 \mathrm{mmHg})$ according to baseline body mass index (BMI)

\begin{tabular}{lllllll}
\hline Patient groups & Normal BMI group & Overweight group & $\boldsymbol{P}$ & Obese group & $\boldsymbol{P}$ & $\boldsymbol{P 1}$ \\
\hline 2 weeks & $17.7 \%$ & $15.6 \%$ & NS & $13.6 \%$ & NS & NS \\
1 month & $52.0 \%$ & $47.1 \%$ & $<0.05$ & $42.1 \%$ & $<0.01$ & 0.0001 \\
3 months & $81.8 \%$ & $78.1 \%$ & NS & $70.9 \%$ & 0.0001 & 0.0001 \\
\hline
\end{tabular}

$P$, comparison between overweight or obese vs. normal BMI group

$P 1$, comparison between obese vs. overweight group

Table 5 Creatinine levels and estimated value of the glomerular filtration rates (GFR) at baseline and after the treatment, according to baseline body mass index (BMI)

\begin{tabular}{lllllll}
\hline Parameter & Normal BMI group & Overweight group & $\boldsymbol{P}$ & Obese group & $\boldsymbol{P}$ & $\boldsymbol{P 1}$ \\
\hline \multicolumn{2}{l}{$\begin{array}{l}\text { Creatinine, } \mu \mathrm{mol} / \mathrm{L} \\
\text { Baseline }\end{array} \quad 85.6 \pm 0.4$} & $85.7 \pm 0.3$ & NS & $88.1 \pm 0.3$ & 0.0001 & 0.0001 \\
$\begin{array}{l}\text { 3 months } \\
\text { GFR (CKD-EPI), } \mathrm{ml} / \mathrm{min} / 1.73 \mathrm{~m}^{2}\end{array}$ & $81.2 \pm 0.3$ & NS & $83.9 \pm 0.4$ & 0.0038 & 0.0001 \\
Baseline & $73.9 \pm 2.7$ & $74.3 \pm 1.5$ & NS & $69.4 \pm 0.9$ & 0.0001 & 0.0001 \\
3 months & $77.4 \pm 1.3$ & $77.8 \pm 2.8$ & NS & $72.3 \pm 1.1$ & NS & NS \\
\hline
\end{tabular}

Data presented are mean $\pm \mathrm{SE}$

GFR glomerular filtration rates, CKD-EPI formula for calculation of glomerular filtration rate

$P$, comparison between overweight or obese vs. normal BMI group

$P 1$, comparison between obese vs. overweight group

\section{DISCUSSION}

This post hoc analysis of the FORSAGE study provides important data on the cardiovascular risk profile of patients treated for hypertension in routine clinical practice in the Russian Federation by BMI category, and the influence that this may have on treatment efficacy. There was a higher incidence of concomitant diseases associated with the metabolic syndrome among overweight and obese participants, including diabetes mellitus, higher BP levels, serum cholesterol and creatinine, and lower GFR. When patients with BP uncontrolled on their previous antihypertensive regimen were switched to perindopril arginine/indapamide SPC, statistically significant reductions in $\mathrm{BP}$ and higher rates of target BP achievement were observed in all BMI groups. At M3, over $70 \%$ of patients treated with the SPC had achieved target $\mathrm{BP}$, including overweight and obese subjects who had the highest BP at baseline.

Worldwide, nearly a third of the population is now classified as overweight or obese [9]. In the current study, $48.7 \%$ of patients had a BMI in the overweight range, while $34.7 \%$ were obese. Similar findings have been observed in a recent large epidemiological study from the Russian Federation, where the prevalence of BMI-defined obesity was estimated as 33.4\% [10]. In addition to hypertension, overweight is associated with multiple CVD risk factors, including hypercholesterolemia, diabetes, and metabolic syndrome. Pooled data from a number of US longitudinal population-based cohort studies have been used to estimate lifetime risk estimates of CVD by weight status [11]. The results showed that overweight and obesity 
were associated with earlier onset of CVD, and a greater proportion of life lived with CVD morbidity. Obesity was additionally associated with shorter overall survival compared with adults with normal BMI.

The FORSAGE findings are in line with previously reported studies. The Global Cardiometabolic Risk Profile in patients with Hypertension Disease (GOOD) survey, which included 3370 patients from 12 European countries, showed that patients with uncontrolled BP had a higher BMI, greater waist circumference, and other impaired metabolic parameters (increased fasting plasma glucose, total cholesterol, and triglyceride levels), as well as lower levels of high-density lipoprotein (HDL) cholesterol in women [12]. In the GOOD survey, the prevalence of metabolic syndrome (ATP III criteria) was significantly higher in patients with uncontrolled versus controlled BP (66.5 vs. $35.5 \%$, respectively). Multivariate analysis revealed that the metabolic syndrome was associated with a 2.6-fold greater likelihood of poor BP control [12]. Further analysis of the GOOD survey reported that it was the visceral obesity and dyslipidemia components of the metabolic syndrome that were associated with the poor response to antihypertensive treatment and not impaired glucose tolerance [13].

Regardless of BMI group, switching from previous therapy with ACEI, ARB, and diuretics to SPC perindopril arginine/indapamide at full dose $(10 \mathrm{mg} / 2.5 \mathrm{mg})$ was associated with statistically significant decreases in BP and higher rates of target BP achievement (despite the fact that most patients had been receiving combination therapy including renin-angiotensin-aldosterone system (RAAS) inhibitors and diuretics at baseline). However, although the overall proportion of patients achieving target BP was quite high (70-80\%), fewer obese subjects achieved target BP compared with subjects in the overweight and normal BMI groups at all study time points.

The reduced efficacy of antihypertensive therapy in obese subjects may relate to the fact that hypertension in these individuals has different pathogenetic features [14]. In people with obesity, the kidneys are subject to outer compression by intra-abdominal adipose tissue and increased intra-abdominal pressure, as well as inner compression of renal medullary sinuses by penetrating adipose tissue and increased intrarenal tissue compression. The result is impaired blood supply to the kidneys and subsequent activation of the renin-angiotensin and sympathetic nervous systems [15]. The adipose tissue itself also plays an important role in the activation of RAAS. Individual adipocytes have all the components of the RAAS, and an accumulation of fat mass leads to an increase in angiotensinogen followed by increased angiotensin II levels, higher expression of angiotensin II type 1 receptors, and aldosterone production. This causes a significant change in the structural and functional state of the cardiovascular system (left ventricular hypertrophy, vascular remodeling, and endothelial dysfunction) on the one hand, and an increase in salt sensitivity and an increase in the reabsorption of sodium and fluid on the other, with a resultant increase in fluid volume [16].

Adipocytes are hormonally active cells that trigger the production of leptin, adipsin, adiponectin, and proinflammatory cytokines (TNF$\alpha$, interleukins, transforming growth factor- $\beta$, and monocyte chemoattractant protein 1), and the release of fatty acids [17]. The fasting level of leptin secreted by adipocytes becomes higher with increased grade of obesity. At the same time, leptin increases the activity of the sympathetic nervous system, particularly in the kidneys. The consequences of sustained sympathetic overdrive include increases in cardiac output and heart rate, tubular reabsorption of sodium, and intravascular blood volume [18]. The combination of perindopril and indapamide therefore acts on the major mechanisms involved in obesity-related hypertension: adipocyte-associated RAAS activation (inhibited by perindopril) and distal tubule sodium reabsorption (blocked by indapamide).

Greater attention is now also being paid to the association between oxidative stress and hypertension in obese patients. Oxidative stress and increased production of reactive oxygen species in glomerular podocytes, endothelial cells, and mesangial cells are known to lead to the development and progression of kidney damage in metabolic disorders [19]. Increased 
arterial stiffness and early return of reflected waves represent a rise in central systolic BP, a drop in diastolic BP, and an increase in pulse pressure, and mediate at least some of the effects of CKD on cardiac function [20]. Arterial stiffness can be improved by a few classes of antihypertensive drugs, such as ACEI, ARB, and direct renin inhibitors. These are able to reduce arterial stiffness independently of BP changes, which may explain the effectiveness of the fixed-dose combination of perindopril/indapamide in this category of patient. It was noted that the incidence of glomerulopathy associated with obesity increased tenfold between 1986 and 2000 [21]. Obesity is considered to be a powerful factor in the initiation and progression of chronic kidney disease [22, 23]. Importantly, in the FORSAGE study, perindopril/ indapamide treatment was not associated with acute increases in serum creatinine or a sudden decline in GFR. The combination was well tolerated, with adverse events in line with those reported in previous studies in a variety of patient groups [24-26].

The complex pathogenesis of obesity-related hypertension highlights the need for specific pharmacological recommendations in the form of combination antihypertensive therapy [27]. Subgroup analysis of obese patients from the large randomized ASCOT BPLA (Anglo-Scandinavian Cardiac Outcomes Trial, Blood Pressure Lowering Arm) trial, suggests that drugs such as thiazide diuretics and ACEI are superior for preventing cardiovascular events in obesity-related hypertension [28], and it has been suggested that this combination should be considered as first-line antihypertensive drug therapy in obesity [29]. In the FORSAGE study, patients were initially receiving combination therapy, but it is likely that the specific components of the combination therapy regimen may provide an explanation for the differences in the efficacy of treatment.

No significant difference was observed between the mean number of drugs taken by patients with normal BMI (3.9) and by overweight subjects (4.1), but obese patients were using statistically more drug classes (4.4; $P=0.0001)$. The proportion of patients taking CCB was low $(13.4,16.1$, and $16.1 \%$ in the normal, overweight, and obese BMI groups, respectively), and as these numbers did not change during the study, CCB use could not have influenced the antihypertensive effect.

There is evidence that perindopril leads to a more pronounced decrease in BP than other ACEI in most patients [23] and greater BP-lowering effect than with the ARB, particularly in obesity [30]. In patients with a large number of cardiovascular risk factors such as in the current study, the effect of perindopril is reported to be even more pronounced [31]. Perindopril, in contrast to ARB such as losartan, also increases insulin sensitivity in overweight patients [32]. Furthermore, recent data indicate that a fixed combination of perindopril and indapamide not only leads to a decrease in BP in patient groups achieving and not achieving target BP on preceding therapy, but also to decreases in leptin levels of 14.4 and $10.0 \%$, and high-sensitivity C-reactive protein by 17.7 and $11.0 \%$, and an increase in adiponectin levels of 6.7 and $9.9 \%$, respectively $(P<0.01)[15]$.

The switch to a perindopril/indapamide SPC led to an increase in the number of patients taking a diuretic, a required and pathogenetically substantiated component of treatment for obesity-related hypertension. The observed beneficial effect with the perindopril/indapamide SPC may also be partially explained by the replacement of hydrochlorothiazide, which is often used for the treatment of hypertension, with indapamide. Indapamide is considered a preferred drug for obesity-related hypertension because of its marked antihypertensive effect, as well as multiple organ-protective properties [16-18, 33].

Given the increasing rates of overweight and obesity, substantial improvements in CVD risk factor treatment and control rates among obese individuals are needed to prevent future cardiovascular events. Weight loss remains important in patients with obesity-associated hypertension to reduce circulating leptin, decrease sympathetic activation, and improve $\mathrm{BP}$ and other risk factors, but weight loss as a result of lifestyle changes is often modest and difficult to maintain. When treating people with obesity, careful attention should be paid to the selection of appropriate antihypertensive 
therapy. The current study suggests that this should more systematically be brought to the attention of physicians, as in clinical practice, BMI levels in the overweight or obese range appear to have little impact on the type of antihypertensive drugs prescribed. There is currently a lack of clinical trial data evaluating interventions in obese subgroups to determine whether obesity-specific treatments would result in decreased CVD outcomes. No specific recommendations for high BP management in patients with excess weight are discussed in international guidelines, and until the results of randomized clinical trials become available, observational studies such as FORSAGE will continue to inform physicians on the optimal management of their patients.

\section{Study Limitations}

In addition to the limitations regarding the study design (open-label, observational, nonrandomized, lack of control group) and post hoc analysis, limitations of the study include the fact that adherence was not assessed in the post hoc analysis, and LVH measurement techniques were not unified. Only descriptive statistics were used, and there was no calculation of sample size. In addition, as the study reflected routine clinical practice, patients could receive concomitant therapy, which may have introduced bias.

\section{CONCLUSIONS}

Rates of treatment and control of hypertension are suboptimal, particularly among overweight and obese individuals. The mechanisms of action of perindopril and indapamide may make them particularly suitable for use in obesity-associated hypertension. In the present analysis of the observational FORSAGE study, patients not achieving BP targets on previous antihypertensive therapy were switched to an adapted-dose, perindopril arginine $10 \mathrm{mg} / \mathrm{inda}-$ pamide $2.5 \mathrm{mg}$ SPC. At M3, statistically significant reductions in $\mathrm{BP}$ and high rates of target $\mathrm{BP}$ achievement were observed in all patient BMI groups. This included the large proportion of overweight and obese patients, a group particularly difficult to control due to specific pathogenetic mechanisms and additional comorbidities and risk factors.

\section{ACKNOWLEDGEMENTS}

Funding. Sponsorship for this study was provided by Servier, Moscow, Russian Federation. The journal's Rapid Service Fee was funded by Servier, France.

Editorial Assistance. Editorial assistance was provided by Jenny Grice, a freelance medical writer, and funded by Servier, France.

Authorship. All named authors meet the International Committee of Medical Journal Editors (ICMJE) criteria for authorship for this article, take responsibility for the integrity of the work as a whole, and have given their approval for this version to be published.

Disclosures. Maria G. Glezer was a scientific coordinator of this study and received honoraria for lectures from Servier.

Compliance with Ethics Guidelines. The program was approved at the meeting of the Inter-University Ethical Committee. Protocol No. 06-14 dated 20.06.2014 (Moscow). The study was performed in accordance with the International Council for Harmonisation of Technical Requirements for Pharmaceuticals for Human Use (ICH) guidelines for good clinical practice and the ethical principles derived from the revised Declaration of Helsinki. All patients provided written informed consent.

Data Availability. The data sets generated and/or analyzed for this study are not publicly available, as they include medical records of patients from a secondary source.

Open Access. This article is distributed under the terms of the Creative Commons Attribution-NonCommercial 4.0 International License (http://creativecommons.org/licenses/ 
by-nc/4.0/), which permits any non-commercial use, distribution, and reproduction in any medium, provided you give appropriate credit to the original author(s) and the source, provide a link to the Creative Commons license, and indicate if changes were made.

\section{REFERENCES}

1. Li YT, Wang HH, Liu KQ, Lee GK, Chan WM, Griffiths SM, et al. Medication adherence and blood pressure control among hypertensive patients with coexisting long-term conditions in primary care settings: a cross-sectional analysis. Medicine (Baltimore). 2016;95:e3572.

2. La Sierra A, Oliveras A, Armario P, Lucas S. Control de la presión arterial en pacientes en tratamiento con terapia combinada. Med Clin (Barc). 2015;144: $145-50$

3. Labeit AM, Klotsche J, Pieper L, Pittrow D, Einsle F, Stalla GK, et al. Changes in the prevalence, treatment and control of hypertension in Germany? A clinical-epidemiological study of 50.000 primary care patients. PLoS One. 2012;7:e52229.

4. Chopra I, Kamal KM, Candrilli SD, Kanyongo G. Association between obesity and therapeutic goal attainment in patients with concomitant hypertension and dyslipidemia. Postgrad Med. 2014;126: 66-77.

5. Schmieder RE, Gitt AK, Koch C, Bramlage P, Ouarrak $T$, Tschöpe $D$. Achievement of individualized treatment targets in patients with comorbid type- 2 diabetes and hypertension: 6 months results of the DIALOGUE registry. BMC Endocr Disord. 2015;15: 23.

6. Reibis RK, Huber M, Karoff M, Kamke W, Kreutz R, Wegscheider $\mathrm{K}$, et al. Target organ damage and control of cardiovascular risk factors in hypertensive patients. Evidence from the multicenter ESTher registry. Herz. 2015;40(Suppl 2):209-16.

7. Friedberg JP, Rodriguez MA, Watsula ME, Lin I, Wylie-Rosett J, Allegrante JP, et al. Effectiveness of a tailored behavioral intervention to improve hypertension control: primary outcomes of a randomized controlled trial. Hypertension. 2015;65:440-6.

8. Glezer MG, Deev AD. How to increase the effectiveness of antihypertensive therapy in clinical practice: results of the Russian observational program FORSAZH. Kardiologiia. 2016;56:18-24.
9. Chooi YC, Ding C, Magkos F. The epidemiology of obesity. Metabolism. 2019;92:6-10.

10. Zhernakova YV, Zheleznova EA, Chazova IE, Oshchepkova EV, Dolgusheva YA, Yarovaya EB, et al. The prevalence of abdominal obesity and the association with socioeconomic status in Regions of the Russian Federation, the results of the epidemiological study-ESSE-RF. Ter Arkh. 2018;90(10): 14-22.

11. Khan SS, Ning H, Wilkins JT, Allen N, Carnethon M, Berry JD, et al. Association of body mass index with lifetime risk of cardiovascular disease and compression of morbidity. JAMA Cardiol. 2018;3(4):280-7.

12. Kjeldsen SE, Naditch-Brule L, Perlini S, Zidek W, Farsang C. Increased prevalence of metabolic syndrome in uncontrolled hypertension across Europe: the Global Cardiometabolic Risk Profile in Patients with hypertension disease survey. J Hypertens. 2008;26:2064-70.

13. Zidek W, Naditch-Brûlé L, Perlini S, Farsang C, Kjeldsen SE. Blood pressure control and components of the metabolic syndrome: the GOOD survey. Cardiovasc Diabetol. 2009;8:51.

14. Astashkin EI, Glezer MG. Obesity and arterial hypertension [Russian]. Probl Women Health. 2008;4(3):23-33.

15. Nedogoda SV, Chumachek EV, Ledyaeva AA, Tsoma VV, Salasyuk AS, Smirnova VO, et al. Optimization of control of blood pressure, metabolic disorders and target organ protection with fixed perindopril and indapamide combination in treated patients with arterial hypertension. Kardiologiia. 2017;57: 5-11.

16. Roush GC, Ernst ME, Kostis JB, Tandon S, Sica DA. Head-to-head comparisons of hydrochlorothiazide with indapamide and chlorthalidone: antihypertensive and metabolic effects. Hypertension. 2015;65:1041-6.

17. Wang S, Li J, Zhou X, Liu K, Zhang X, Meng Q, et al. Comparison between the effects of hydrochlorothiazide and indapamide on the kidney in hypertensive patients inadequately controlled with losartan. J Hum Hypertens. 2017;31:848-54.

18. DiNicolantonio JJ, Bhutani J, Lavie CJ, O'Keefe JH. Evidence-based diuretics: focus on chlorthalidone and indapamide. Future Cardiol. 2015;11:203-17.

19. Wan C, Su H, Zhang C. Role of NADPH oxidase in metabolic disease-related renal injury: an update. Oxid Med Cell Longev. 2016;2016:7813072. 
20. Zanoli L, Lentini P, Briet M, Castellino P, House AA, London GM, Malatino L, McCullough PA, Mikhailidis DP, Boutouyrie P. Arterial stiffness in the heart disease of CKD. J Am Soc Nephrol. 2019;30(6): 918-28.

21. Kambham N, Markowitz GS, Valeri AM, et al. Obesity-related glomerulopathy: an emerging epidemic. Kidney Int. 2001;59:1498-509.

22. Kovesdy CP, Furth SL, Zoccali C. Obesity and kidney disease: hidden consequences of the epidemic. Blood Purif. 2017;43:346-54.

23. Ionescu DD. Antihypertensive efficacy of perindopril $5-10 \mathrm{mg} /$ day in primary health care: an openlabel, prospective, observational study. Clin Drug Investig. 2009;29:767-76.

24. Kang S, Wu YF, An N, Ren M. A systematic review and meta-analysis of the efficacy and safety of a fixed, low-dose perindopril-indapamide combination as first-line treatment of hypertension. Clin Ther. 2004;26(2):257-70.

25. Mourad JJ, Le Jeune S. Evaluation of high dose of perindopril/indapamide fixed combination in reducing blood pressure and improving end-organ protection in hypertensive patients. Curr Med Res Opin. 2009;25(9):2271-80. https://doi.org/10.1185/ 03007990903186787.

26. Netchessova TA, Shepelkevich AP, Gorbat TV, NIKA Study Group. Efficacy of single-pill perindopril/indapamide in patients with hypertension and type 2 diabetes. High Blood Press Cardiovasc Prev. 2014;21(1):63-9.

27. Carnagarin R, Matthews V, Gregory C, Schlaich MP. Pharmacotherapeutic strategies for treating hypertension in patients with obesity. Expert Opin Pharmacother. 2018;19:643-51.

28. Dahlöf B, Sever PS, Poulter NR, Wedel H, Beevers DG, Caulfield M, ASCOT Investigators, et al. Prevention of cardiovascular events with an antihypertensive regimen of amlodipine adding perindopril as required versus atenolol adding bendroflumethiazide as required, in the Anglo-Scandinavian Cardiac Outcomes Trial-Blood Pressure Lowering Arm (ASCOTBPLA): a multicentre randomised controlled trial. Lancet. 2005;366(9489):895-906.

29. Wenzel UO, Benndorf R, Lange S. Treatment of arterial hypertension in obese patients. Semin Nephrol. 2013;33:66-74.

30. Nedogoda SV, Ledyaeva AA, Chumachok EV, Tsoma VV, Mazina G, Salasyuk AS, et al. Randomized trial of perindopril, enalapril, losartan and telmisartan in overweight or obese patients with hypertension. Clin Drug Investig. 2013;33:553-61.

31. Ogilvie RI, Anand S, Roy P, Souza S. Perindopril for control of blood pressure in patients with hypertension and other cardiovascular risk factors. Clin Drug Investig. 2008;28:673-86.

32. Fogari R, Zoppi A, Lazzari P, Preti P, Mugellini A, Corradi $\mathrm{L}$, et al. ACE inhibition but not angiotensin II antagonism reduces plasma fibrinogen and insulin resistance in overweight hypertensive patients. J Cardiovasc Pharmacol. 1998;32(4):616-20.

33. Semenkin AA, Zhenatov AB, Zhivilova LA, Nechaeva GI, Pritykina TV, Chindareva OI, et al. Direct comparison of endothelial and metabolic effects of perindopril combination with indapamide retard or hydrochlorothiazide. Kardiologiia. 2014;54(11):25-9. 